\title{
Rational Optimization and Imaging In Vivo of a Genetically Encoded Optical Voltage Reporter
}

\author{
Lucas Sjulson ${ }^{1}$ and Gero Miesenböck ${ }^{1,2}$ \\ ${ }^{1}$ Department of Cell Biology, Yale University School of Medicine, New Haven, Connecticut 06520, and 2Department of Physiology, Anatomy, and Genetics, \\ University of Oxford, Oxford OX1 3PT, United Kingdom
}

\begin{abstract}
The hybrid voltage sensor (hVOS) combines membrane-targeted green fluorescent protein and the hydrophobic anion dipicrylamine (DPA) to provide a promising tool for optical recording of electrical activity from genetically defined populations of neurons. However, large fluorescence signals are obtained only at high DPA concentrations $(>3 \mu \mathrm{M})$ that increase membrane capacitance to a level that suppresses neural activity. Here, we develop a quantitative model of the sensor to guide its optimization and achieved an approximate threefold increase in fractional fluorescence change at a lower DPA concentration of $2 \mu \mathrm{M}$. Using this optimized voltage reporter, we perform optical recordings of evoked activity in the Drosophila antennal lobe with millisecond temporal resolution but fail to detect action potentials, presumably because spike initiation and/or propagation are inhibited by the capacitive load added even at reduced DPA membrane densities. We evaluate strategies for potential further improvement of hVOS quantitatively and derive theoretical performance limits for optical voltage reporters in general.
\end{abstract}

Key words: neuroimaging; membrane potential; protein-based sensor; fluorescence; multiphoton microscopy; membrane capacitance

\section{Introduction}

Understanding the organization and function of neural circuits would benefit greatly from experimental tools that permit detailed characterization of local circuit dynamics. The ideal technique would detect electrical signals in single cells with millisecond temporal resolution and sample densely a significant fraction of identified neurons in a given spatial region. Multielectrode arrays are generally capable of high spatial and temporal resolution (Kralik et al., 2001) but not high-density sampling, although progress is being made on this front (Buzsaki, 2004). Calcium imaging has high spatial resolution and can sample at very high densities (Ohki et al., 2005) but fundamentally suffers from low temporal resolution (Smetters et al., 1999), which can be partially ameliorated through deconvolution (Yaksi and Friedrich, 2006). Voltage-sensitive dye imaging meets all of these requirements in some tissue preparations (Salzberg et al., 1973; Obaid et al., 1999), but nonspecific labeling and phototoxicity are significant obstacles in most mammalian systems.

Genetically encoded optical voltage reporters provide a particularly elegant approach to this problem (Siegel and Isacoff, 1997; Miesenböck and Kevrekidis, 2005; Sjulson and Miesenböck, 2007). Most of these reporters combine nonconducting

\footnotetext{
Received Jan. 7, 2008; revised April 10, 2008; accepted April 14, 2008.

This work was supported by National Institutes of Health (NIH) Medical Scientist Training Program Grant GM07739 (L.S.) and by grants from the Katherine Beineke Foundation (L.S.), NIH (G.M.), the Dana Foundation (G.M.), the Office of Naval Research (G.M.), and the Medical Research Council (G.M.). We thank Robert Roorda and members of the Miesenböck laboratory for discussions and technical assistance, B. Chanda and F. Bezanilla for the EGFP-F construct, and D. Piston for the Cerulean construct.

Correspondence should be addressed to Lucas Sjulson at his present address: The Rockefeller University, 1230 York Avenue, \#95, New York, NY 10065. E-mail: lukesjulson@gmail.com.

D0I:10.1523/JNEUROSCI.0055-08.2008

Copyright $\odot 2008$ Society for Neuroscience $\quad$ 0270-6474/08/285582-12\$15.00/0
}

mutants of voltage-gated cation channels with derivatives of the green fluorescent protein (GFP). For example, FlaSh and its variants (Siegel and Isacoff, 1997; Guerrero et al., 2002) are based on the Shaker potassium channel, whereas SPARC (sodium channel protein-based activity reporting construct) (Ataka and Pieribone, 2002) is a mutant $\mathrm{Na}_{\mathrm{v}} 1.4$ sodium channel, and VSFP1 (voltage-sensitive fluorescent protein 1) (Sakai et al., 2001) uses an isolated voltage sensor domain of the Kv2.1 potassium channel.

More recently, Chanda et al. (2005b) introduced a different type of genetically encoded optical voltage reporter. This design, termed hybrid voltage sensor (hVOS), combines a membrane targeted GFP, which serves as a fluorescence resonance energy transfer (FRET) donor, with a voltage-sensitive FRET acceptor dye, dipicrylamine (DPA), that quenches GFP fluorescence. DPA is a hydrophobic anion that partitions into the lipid bilayer and distributes between the inner and outer membrane leaflets according to membrane potential, thus quenching GFP fluorescence in a voltage-dependent manner. hVOS exhibits a high fractional fluorescence change $(\Delta F / F)$ of up to $34 \%$ per $100 \mathrm{mV}$, responds with submillisecond kinetics, and is the only genetically encoded optical voltage reporter that has been used to record neuronal action potentials evoked by current injection without averaging (Chanda et al., 2005b). However, like all voltage reporters that sense membrane potential with mobile probe charges, hVOS increases membrane capacitance significantly. In fact, hVOS only produces a substantial $\Delta F / F$ at high DPA concentrations $(\geq 3 \mu \mathrm{M})$ that increase membrane capacitance to a level where synaptically evoked action potentials are inhibited.

In this paper, we develop a quantitative model of the GFPDPA interaction. This model is used to guide the optimization of hVOS, yielding a $\sim 3$-fold increase in $\Delta F / F$ at low DPA concen- 
trations. The optimized hVOS is expressed in the Drosophila olfactory system, where optical recordings of electrical activity are performed with high temporal resolution. The model is then used to evaluate potential strategies for further improvements of hVOS and derive theoretical performance limits for optical voltage reporters in general.

\section{Materials and Methods}

Chemicals and solutions. All reagents were obtained from Sigma-Aldrich, with the exception of DPA, which was obtained from LaboTest and was stored as a $20 \mathrm{~mm}$ stock solution in DMSO at $-80^{\circ} \mathrm{C}$. For whole-cell voltage-clamp experiments in HeLa and human embryonic kidney 293 (HEK293) cells, solutions were used that block most ionic conductances. The intracellular solution was composed of $130 \mathrm{~mm} \mathrm{~N}$-methyl-Dglucamine (NMDG) gluconate, $10 \mathrm{~mm}$ NMDG chloride, 1 mm EGTA, and $10 \mathrm{~mm}$ HEPES, pH 7.4, raised to $\sim 340 \mathrm{mOsm}$ with sucrose. The extracellular solution was composed of $130 \mathrm{~mm}$ NMDG gluconate, 10 mM NMDG chloride, $2 \mathrm{~mm} \mathrm{MgCl}_{2}$, and $10 \mathrm{~mm}$ HEPES, pH 7.2, raised to $\sim 320$ mOsm with sucrose. Odor and suction electrode stimulation experiments in flies were performed using adult hemolymph with $2 \mathrm{~mm}$ $\mathrm{Ca}^{2+}$ (Wong et al., 2005).

Molecular biology. Experiments in mammalian cells were performed using the pCI-neo expression vector (Promega), which contains the neomycin phosphotransferase gene, allowing selection of stable cell lines. All GFP variants were PCR amplified and inserted into pCI-neo using EcoRI and NotI restriction sites, verified by sequencing, and subcloned into the pUAST vector used for the generation of transgenic flies. PCR templates were either codon-optimized enhanced GFP (EGFP) (Clontech), codonoptimized EGFP-F [generously provided by B. Chanda (University of Wisconsin, Madison, WI) and F. Bezanilla (University of Chicago, Chicago, IL)], or Cerulean (generously provided by D. Piston, Vanderbilt University Medical Center, Nashville, TN) (for full sequences and details of plasmid construction, see supplemental material, available at www.jneurosci.org).

Cell culture and transfection. HeLa and HEK293 cells were maintained in Advanced DMEM (Invitrogen) supplemented with 2.5\% fetal bovine serum, $2 \mathrm{~mm}$ GlutaMAX (Invitrogen), $100 \mathrm{U} / \mathrm{ml}$ penicillin, and 100 $\mu \mathrm{g} / \mathrm{ml}$ streptomycin at $37^{\circ} \mathrm{C}$ in $5 \% \mathrm{CO}_{2}$. For stable transfection, cells were grown to $50-70 \%$ confluency in six-well plates, transfected using FuGene 6 transfection reagent (Roche Diagnostics) according to the manufacturer instructions, and selected in $600 \mu \mathrm{g} / \mathrm{ml}$ G418. Stable transfectants were further screened for adequate GFP expression using epifluorescence microscopy.

Transgenic Drosophila. EGFP constructs in the pUAST vector (Brand and Perrimon, 1993) were injected by BestGene, and transgenic flies were isolated using standard techniques. For antennal lobe expression, two copies of UAS-EGFP- $\triangle$ CAAX, UAS-PS-EGFP- $\triangle$ CAAX, or UASsynapto-pHluorin (Miesenböck et al., 1998; Ng et al., 2002) were used with either one or two copies of OR83b-GAL4 (Ng et al., 2002).

Wide-field fluorometry. Wide-field fluorometry was performed on a Carl Zeiss Axioskop microscope with a $40 \times$ water immersion objective [numerical aperture (NA), 0.8] using a Hamamatsu R1527P photomultiplier tube in a D-104 microscope photometer with a model 804 photomultiplier detection system (Photon Technology International). The output of the photometer was lowpass filtered at $1 \mathrm{kHz}$ and sampled at 20 $\mathrm{kHz}$, the same sampling rate as the electrophysiology channels. For voltage-clamp fluorometry, cells were illuminated by a Carl Zeiss HBO100 arc lamp, using either an EGFP or enhanced cyan fluorescent protein (ECFP) filter set (Chroma Technology). For suction electrode stimulation experiments, the sample was illuminated by a Carl Zeiss HBO100 W/2 arc lamp powered by a Carl Zeiss high-stability power supply, attenuated by a $50 \%$ neutral density filter. Despite the high stability power supply, power spectral estimates showed clear peaks at 120 and $240 \mathrm{~Hz}$. These were subtracted off-line in Matlab 7 (Mathworks) using multitaper estimation of a spectral line in a colored noise background (Thomson, 1982).

Two-photon line scan fluorometry. A previously described high-speed two-photon laser scanning microscope (Roorda et al., 2004) was modi- fied to enable line scanning. Briefly, an analog circuit was created to provide a command signal to the mirror galvanometer controlling the beam position in the $\mathrm{Y}$ dimension, enabling rapid computer-controlled switching between frame scan and line scan modes. In either mode, the beam was scanned in the $\mathrm{X}$ dimension at a rate of $15.8 \mathrm{kHz}$, with 600 pixels in each scan line. Data were analyzed off-line with Matlab. For additional details, see the study by Sjulson (2007).

Voltage-clamp electrophysiology. Voltage clamp was performed in the whole-cell patch configuration using an AxoClamp 2B amplifier with an HS-2A headstage (Molecular Devices). Amplifier outputs were lowpass filtered at $6-8 \mathrm{kHz}$ and sampled at $20 \mathrm{kHz}$. Electrodes were pulled from $1.5 \mathrm{~mm}$ outer diameter (OD) patch pipette glass (World Precision Instruments) and fire-polished to an average tip resistance of 5-7 M $\Omega$. Series resistance and capacitance compensation were not used, because currents were negligible at holding potentials. Data were acquired using pClamp 8.0 (Molecular Devices) and analyzed using Matlab 7 (Mathworks). Cells were held at $-40 \mathrm{mV}$ for $50 \mathrm{~ms}$, stepped to the test potential for $50 \mathrm{~ms}$, and then brought back to $-40 \mathrm{mV}$ for $50 \mathrm{~ms}$. The test potential ranged from -100 to $+50 \mathrm{mV}$ in $10 \mathrm{mV}$ increments.

Suction electrode stimulation. The fly brain was removed from the head capsule, leaving the antennal nerve intact (Wong et al., 2005). Antennal nerve stimulation was performed using a suction electrode pulled from 1 $\mathrm{mm}$ OD borosilicate glass (A-M Systems). The pipette glass was pulled to a large-diameter opening $(\sim 60 \mu \mathrm{m})$ and fire-polished to $30-36 \mu \mathrm{m}$. A suction electrode holder (A-M Systems) was used with $\mathrm{Ag} / \mathrm{AgCl}$ wires, and electrical pulses were administered using an A365 constant current stimulation isolator (World Precision Instruments). For hVOS experiments, the stimulus consisted of 150 pulses of $1 \mathrm{~ms}$ duration and $100 \mu \mathrm{A}$ amplitude with alternating polarity, whereas for synapto-pHluorin experiments, the stimulus consisted of trains of 101 -ms pulses of alternating polarity at $3 \mathrm{~ms}$ intervals. The $\mathrm{PH} 1$ heated recording chamber (Warner Instruments) was heated to $25^{\circ} \mathrm{C}$ using a modified Series 800 thermoelectric cooler controller (Alpha Omega Instrument).

\section{Results \\ Model of hVOS}

A simple model (Fig. 1A) forms the basis for our rational optimization of hVOS. We assume that GFP and DPA molecules are uniformly randomly distributed along a planar lipid membrane. The distance DPA translocates between the two membrane leaflets is $30 \AA$, and each GFP chromophore is assumed to be held at a fixed distance $y$ of $30 \AA$ from the inner membrane leaflet (see below for a discussion of this assumption). At any given time, only a small fraction of GFP molecules is in the excited state; as a result, saturation of DPA-mediated quenching is negligible, and the statistical behavior of one GFP molecule over many trials is equivalent to that of many GFP molecules in one trial (for discussion, see supplemental material, available at www.jneurosci. org). Other parameters of the model include the calculated Förster distance $R_{0}$ of $37 \AA$ for the GFP-DPA pair (Chanda et al., $2005 \mathrm{~b}$ ); at this distance, the efficiency of energy transfer is $50 \%$. Effects of transition dipole orientation are neglected, including effects of rotational diffusion. Lateral diffusion is assumed to be negligible during the fluorescence lifetime of GFP, which is $\sim 2.9$ ns (Swaminathan et al., 1997). An action potential is treated as a square pulse from $V_{m}=-70 \mathrm{mV}$ to $V_{m}=+30 \mathrm{mV}$, and the proportion of DPA molecules in the inner versus outer membrane leaflets follows a Boltzmann distribution with parameters $z \approx-1$ and $V_{0} \approx-55 \mathrm{mV}$ as follows:

$$
\frac{p_{\text {in }}}{p_{\text {out }}}=\exp \left(\frac{-z F\left(V_{m}-V_{0}\right)}{R T}\right) .
$$

In practice, $z$ and $V_{0}$ vary between different cells and cell types, with reported values of $z$ ranging from -0.63 in squid axon (Fernandez et al., 1983) to approximately -1 in Xenopus oocytes 
(Chanda et al., 2005a); in mouse myocytes, $z$ is between -0.6 and -1 (DiFranco et al., 2007). The effect of this variation is discussed below (see Fig. 5).

Previous studies have determined the membrane density of DPA from capacitance measurements (Fernandez et al., 1983 ) and found that the relationship between nominal DPA concentration in the bath and DPA density in the membrane is complex, depending on the lipid composition of the bilayer, tissue penetration, and saturation effects at higher concentrations (Wang and Bruner, 1978). For our purposes, we assume that the DPA membrane density $A_{T}$ is linearly proportional to the DPA bath concentration, with 8300 DPA molecules $/ \mu \mathrm{m}^{2}$ corresponding to $2 \mu \mathrm{M}$ DPA in the bath, as calculated by Chanda et al. (2005b).

Efficiency of FRET between GFP (donor) and DPA (acceptor) is equal to $1-Q$, where $Q$ is the relative quantum yield, or the ratio of donor fluorescence with acceptors present to donor fluorescence in the absence of acceptors. $Q$ is a sixth-order nonlinear function of the distance, $r$, between GFP and DPA as follows:

$$
Q=\frac{1}{1+\sum_{i} \frac{R_{0}{ }^{6}}{r_{i}^{6}}},
$$

where $r_{i}$ denotes the distance between GFP and the $i$ th DPA molecule, and $R_{0}$ denotes the Förster radius, the distance at which one DPA molecule will quench 50\% of GFP fluorescence (i.e., $Q=0.5$ ). Qualitatively, $Q$ decreases rapidly as a DPA molecule moves inside of the Förster radius $R_{0}$, to a value of 0 (no fluorescence) when $r \ll R_{0}$. Likewise, $Q$ increases rapidly as a DPA molecule moves outside of the Förster radius, to a value of $Q=1$ (full fluorescence) when $r \gg$ $R_{0}$, meaning that interactions with DPA molecules far beyond $R_{0}$ are not significant. Over realistic ranges of fluorophore excitation, $Q$ is related to $F$, the total fluorescence collected, as follows:

$$
F=Q F_{\mathrm{PM}}+F_{\mathrm{NS}}
$$

where $F_{\mathrm{PM}}$ represents GFP fluorescence from the plasma membrane, and $F_{\mathrm{NS}}$ represents nonspecific background fluorescence, resulting from autofluorescence and intracellular GFP not associated with the plasma membrane. $\Delta Q / Q$ thus represents an upper bound on $\Delta F / F$ but becomes a valid estimate of $\Delta F / F$ when background fluorescence $F_{\mathrm{NS}}$ is small. Equations 2 and 3 together suggest that $\Delta F / F$ will be highest when $\Delta Q$ is large, namely, when the DPA molecules can be brought from a distance further than $R_{0}$ at hyperpolarized potentials to a distance closer than $R_{0}$ at depolarized potentials.

The primary drawback of hVOS is that $\Delta F / F$ is high only when the density of DPA in the membrane is higher than neurons will tolerate. To understand the requirement for high DPA densities, we compare two models: the "full model," based on the study by Fung and Stryer (1978), takes all DPA molecules in both membrane leaflets into account, whereas the "reduced model" consid-
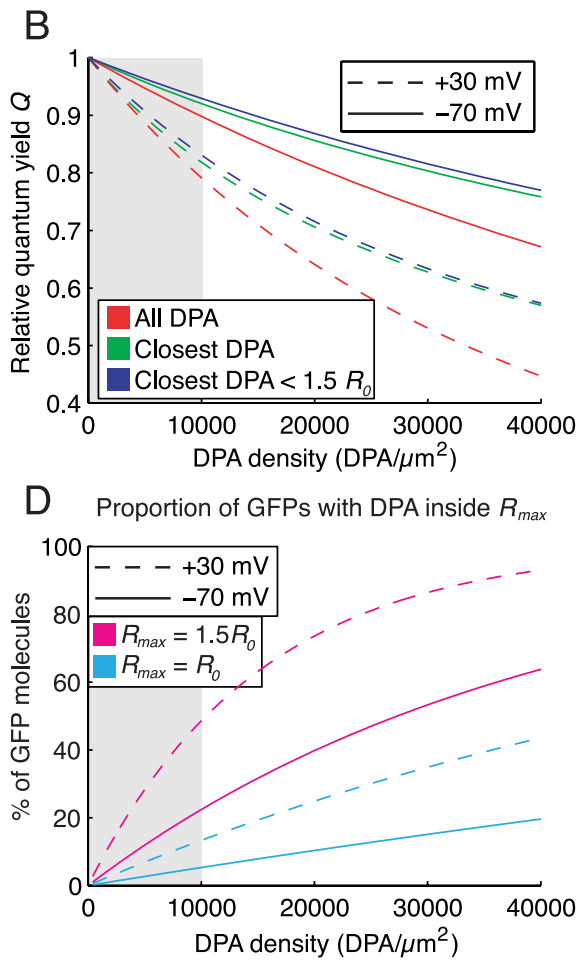

Closest DPA

Closest DPA $<1.5 R_{0}$

$20000 \quad 30000 \quad 40000$

$\mathrm{y}\left(\mathrm{DPA} / \mu \mathrm{m}^{2}\right)$

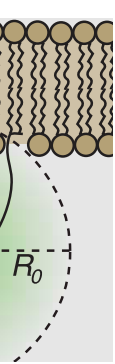

DPA density (DPA $\left.\mu \mathrm{m}^{2}\right)$

Figure 1. GFP fluorescence quenching at physiological DPA concentrations is mediated predominantly by interaction with the closest DPA molecule. $\boldsymbol{A}$, Diagram of GFP-DPA interaction model. $\boldsymbol{B}$, Quenching of GFP is inefficient at physiologically concentrations. At $+30 \mathrm{mV}$, less than half of the GFP molecules are within $1.5 R_{0}$ of a DPA molecule (magenta line), whereas $<14 \%$ have a DPA molecule inside $R_{0}$ (cyan line).

ers only the nearest DPA molecule in the inner membrane leaflet, provided that molecule is closer than a given distance $R_{\max }$ (supplemental material, available at www.jneurosci.org). The models yield similar values of $Q$ for physiologically tolerated DPA concentrations, suggesting that the majority of GFP quenching is mediated by interactions with the single nearest DPA molecule, and the contribution of the nearest DPA molecule is very small if it is outside a radius of $1.5 R_{0}$ (Fig. $1 B$ ).

We next examine $\Delta Q / Q$ for a $100 \mathrm{mV}(-70$ to $+30 \mathrm{mV})$ step as a function of DPA membrane density (Fig. $1 C$ ). At tolerable DPA concentrations, estimates based on the closest DPA molecule approximate the full model well. At higher concentrations, however, the approximation becomes less accurate, suggesting that interactions with multiple DPA molecules in both membrane leaflets become significant. These predictions match experimental observations (Fig. 2D) relatively well, keeping in mind that $\Delta Q / Q$ represents an upper bound on $\Delta F / F$.

Because the majority of quenching is mediated by interactions with DPA molecules inside a radius of $1.5 R_{0}$, we determined the probability of a GFP having a DPA molecule inside a given distance $R_{\max }$ as follows:

$$
P\left(r \leq R_{\max }\right)=1-\exp \left(-p_{\text {in }} A_{T} \pi\left(R_{\max }^{2}-y^{2}\right)\right),
$$

where $A_{T}$ is the density of DPA in the membrane, and $y$ is the GFP-membrane distance (Fig. $1 A$ ). At $+30 \mathrm{mV}$ and DPA densities of $<10,000 \mathrm{DPA} / \mu \mathrm{m}^{2}(\sim 2.4 \mu \mathrm{M})$, less than half of the GFP molecules have a DPA molecule inside the "effective distance" of 


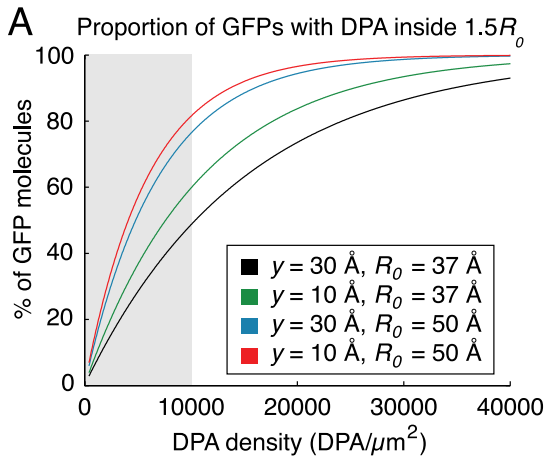

C
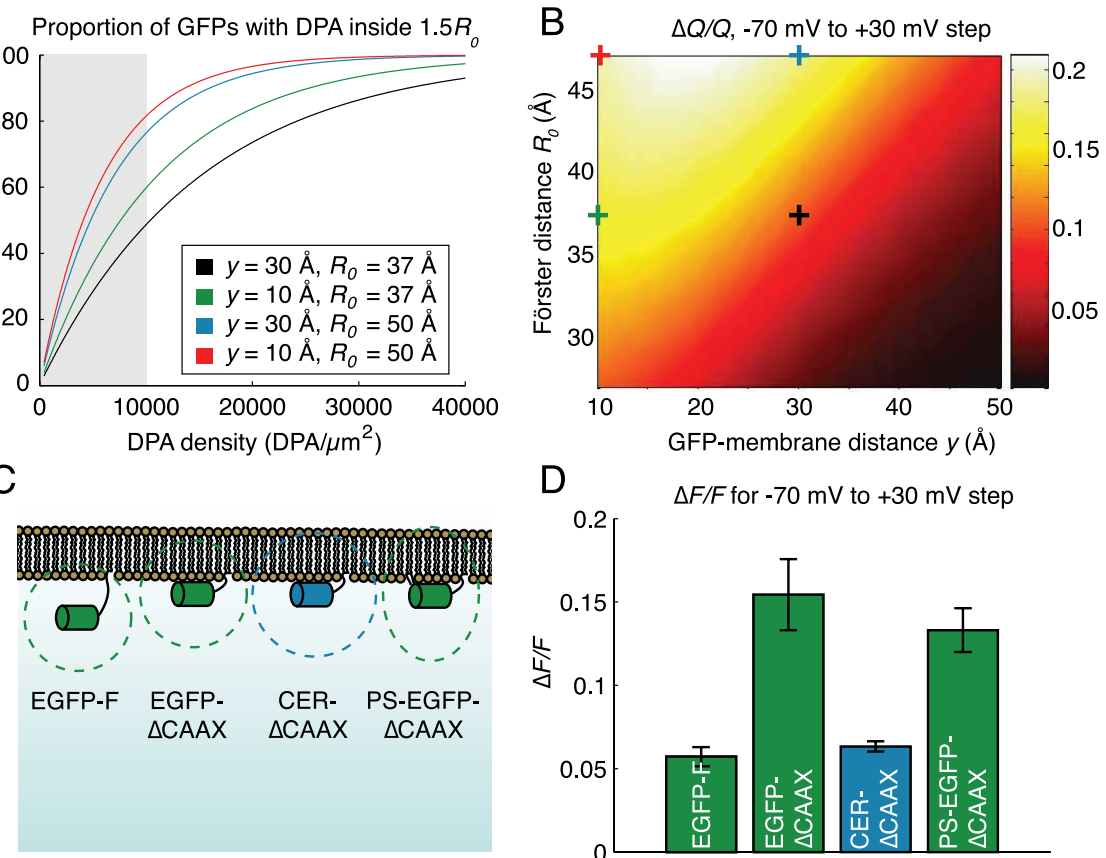

E

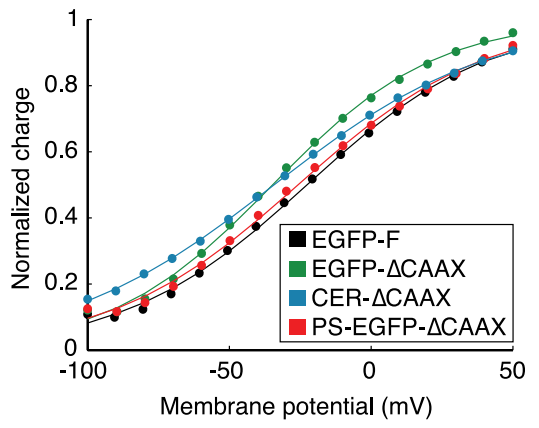

$\mathrm{D}$

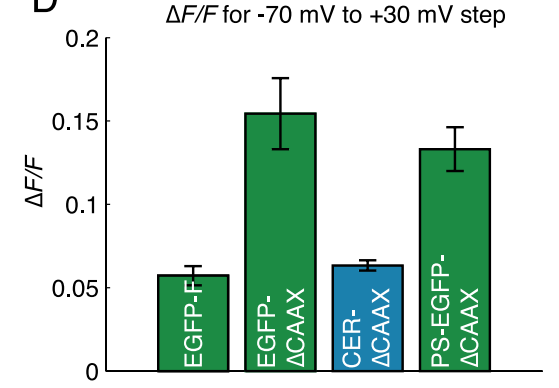

$\mathrm{F}$

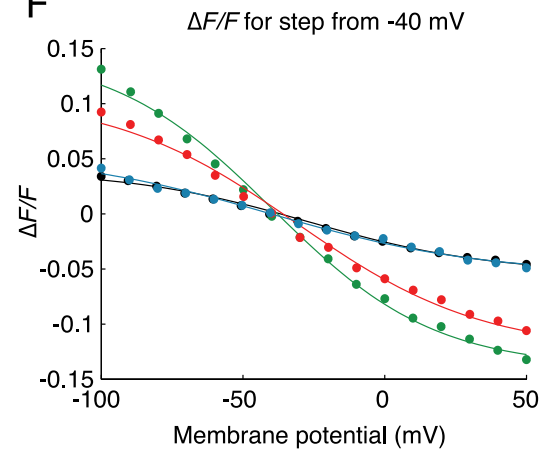

Figure 2. Model-based optimization of hVOS. $A$, Increasing the Förster distance, $R_{0}$, or decreasing the GFP-membrane distance, $y$, substantially increases the proportion of GFP molecules that encounter a DPA molecule inside a radius of $1.5 R_{0}$ and are therefore eligible for quenching. $B$, Calculated values of $\Delta Q / Q$ for a $100 \mathrm{mV}$ step from -70 to $+30 \mathrm{mV}$ at a density of $8300 \mathrm{DPA}$ molecules $/ \mu \mathrm{m}^{2}$ ( $\sim 2 \mu \mathrm{m}$ in bath). Modifying either $R_{0}$ or $y$ can lead to significant changes in $\Delta Q / Q$. C, Constructs tested. EGFP-F is the original hVOS construct, whereas EGFP- $\triangle$ CAAX is designed to minimize $y$. CER- $\triangle$ CAAX has a slightly increased $R_{0}(42 \AA)$ relative to EGFP- $\triangle$ CAAX (37 $\AA$ ), and PS-EGFP- $\triangle$ CAAX contains a palmitoylation site designed to orient the transition dipole moment toward the membrane. $D, \Delta$ F/F per $100 \mathrm{mV}(-70$ to $+30 \mathrm{mV}$ step) as measured by wide-field voltage-clamp fluorometry in $2 \mu \mathrm{M}$ DPA. EGFP- $\Delta$ CAAX and PS-EGFP- $\Delta$ CAAX showed significant increases in $\Delta F / F(p<0.01$; two-tailed $t$ test), whereas CER- $\Delta$ CAAX was not statistically different from EGFP-F $(p=0.24)$. $E$, Experimental measurements of charge translocation in four representative cells (solid dots) are well fit by Equation 1 (solid lines) with $z=-0.80, V_{0}=-23 \mathrm{mV}$ (EGFP-F); $z=-0.90, V_{0}=-35 \mathrm{mV}\left(\right.$ EGFP- $\Delta$ CAAX); $z=-0.68, V_{0}=-34 \mathrm{mV}\left(\right.$ CER- $\Delta$ CAAX); and $z=-0.78, V_{0}=-26 \mathrm{mV}$ (PS-EGFP- $\triangle$ CAAX). $F, \Delta F / F$ for voltage steps from a holding potential of $-40 \mathrm{mV}$ in $2 \mu \mathrm{m}$ DPA. Experimental measurements (solid dots) are well fit by Equation 3 (solid lines) with values of $z$ and $V_{0}$ determined by fitting Equation $1(\boldsymbol{E})$.

$R_{\max }=1.5 R_{0}$, and only $14 \%$ have a DPA molecule inside the Förster distance (Fig. 1D, magenta and cyan dashed lines). In other words, at depolarized potentials, when the GFP should be maximally quenched, only approximately half of the GFP molecules are close enough to a DPA molecule to be "eligible" for quenching, and only $\sim 10 \%$ of the GFP molecules have DPA molecules inside the Förster radius, where the probability of quenching is $>50 \%$. DPA labeling of the membrane at physiologically tolerated concentrations is thus so sparse that most GFP molecules are unlikely to interact with even a single DPA molecule.

\section{Optimization of hVOS:}

model predictions

The main strategy for increasing $\Delta F / F$ at low DPA concentrations is to modify the GFP component of the sensor to enhance its interaction with DPA. This could be accomplished by decreasing the GFPmembrane distance to bring the GFP closer to the DPA molecules and by increasing the Förster distance so that more distant DPA molecules can quench the GFP more effectively.

The GFP-membrane distance, $y$, is determined by the length of the linker sequence between the C-terminal end of the GFP $\beta$-barrel and the prenylated cysteine residue in the CAAX box. Based on the crystal structure of GFP, the last six residues of GFP are unstructured, suggesting that the last residue of the $\beta$-barrel is glycine 232 (Ormö et al., 1996). Counting from this residue, the length of the linker in the EGFP-F construct used by Chanda et al. (2005b) is 27 residues. Estimating the average distance of the EGFP from the membrane is a surprisingly complex matter: detailed simulations and analytical calculations indicate that volume exclusion effects exert an entropic "stretching force," which pulls the GFP further from the membrane than would be expected (Segall et al., 2006; Windisch et al., 2006). Windisch et al. (2006) performed a simulation of a 32 amino acid linker tethered between an infinite wall and a spherical bead of variable diameter. In their simulations, a ball of $30 \AA$ diameter [reasonably close to GFP, which is $\sim 30$ by $40 \AA$ (Ormö et al., 1996; Yang et al., 1996)] leads to an average distance of $\sim 58$ $\AA$ from the attachment point to the center of the ball. We thus estimate the mean distance from the GFP chromophore to the membrane (not the attachment point) to be $\sim 30$ A. If it were possible to place the GFP in direct contact with the membrane, shortening this length to $\sim 10 \AA$, the probability of at least one DPA molecule being closer than $R_{\max }=1.5 R_{0}\left(V_{m}\right.$ $\left.=+30 \mathrm{mV} ; A_{T}=10,000 \mathrm{DPA} / \mu \mathrm{m}^{2}\right)$ increases from 0.49 to 0.60 (Fig. $2 A$ ), and the predicted $\Delta F / F$ from the full model also increases significantly (Fig. $2 B$ ).

Lengthening the Förster distance, $R_{0}$, is the second potential strategy for enhancing GFP-DPA interactions. Increasing $R_{0}$ from 37 to $50 \AA$ has an effect similar to decreasing the GFPmembrane distance to $\sim 10 \AA$ : the probability of a DPA molecule being closer than $R_{\max }=1.5 R_{0}$ increases to 0.76 (Fig. $2 A$ ), and the $\Delta F / F$ predicted from the full model increases substantially (Fig. $2 B$ ).

\section{Optimization of hVOS: experimental results}

Several EGFP constructs were used to test these predictions experimentally (Fig. 2C). In one construct, which we term EGFP- 
$\triangle$ CAAX (Roorda et al., 2004), the 31 C-terminal residues of EGFP-F (which include residues 233-238 of EGFP) were replaced with the last $10 \mathrm{C}$-terminal residues of h-ras, shortening the membrane linker from 27 residues to six. As shown in Figure 2D, the $\Delta F / F$ per $100 \mathrm{mV}$ for EGFP- $\Delta$ CAAX is approximately threefold larger than that of the original EGFP-F at $2 \mu \mathrm{M}$ DPA, a concentration that is sufficiently low for cultured rat hippocampal neurons to fire action potentials.

Increasing the Förster distance $R_{0}$, however, is less straightforward. $R_{0}$ is proportional to the sixth root of four parameters as follows:

$$
R_{0}=9.79 \times 10^{3}\left(\phi_{d} n^{-4} J \kappa^{2}\right)^{1 / 6} \AA
$$

These are $\phi_{d}$, the fluorescence quantum yield of GFP; $n$, the refractive index of the medium; $J$, the overlap of the GFP emission spectrum with the DPA absorption spectrum; and $\kappa^{2}$, the relative orientation parameter. The fluorescence quantum yield of GFP and the refractive index of the medium are difficult, if not impossible, to change, leaving the spectral overlap $J$ and the relative orientation $\kappa^{2}$.

Spectral overlap can be targeted by using GFP mutants with altered emission spectra. One such mutant, Cerulean, contains the ECFP mutations F64L/S65T/Y66W plus additional mutations S72A/Y145A/H148D, which shift the emission peak of the protein to $475 \mathrm{~nm}$ (Rizzo et al., 2004). Factoring in the changes in spectral overlap and slightly increased quantum yield of Cerulean $\left(\phi_{d}=0.62\right)$ relative to EGFP $\left(\phi_{d}=0.60\right)$, the estimated Förster distance is $\sim 42 \AA$ rather than $37 \AA$. As shown in Figure $2 B$, this slight increase in $R_{0}$ is not predicted to yield a significant increase in $\Delta F / F$. In fact, Cer- $\Delta$ CAAX, which contains Cerulean instead of EGFP in an otherwise identical construct, displayed significantly reduced voltage sensitivity relative to EGFP- $\triangle$ CAAX (Fig. $2 D$ ). The decreased $\Delta F / F$ likely reflects increased background signal and cellular autofluorescence in the ECFP emission band.

$\kappa^{2}$ depends on the relative orientations of the transition dipole moments of GFP and DPA. Under conditions of unconstrained rotational diffusion that is rapid relative to the fluorescence lifetime of GFP, $\kappa^{2}=2 / 3$. However, the membrane anchor of the GFP and the association of DPA with the polar phospholipid head groups most likely place constraints on the rotational mobility of these molecules, restricting the transition dipole moments to a preferred distribution of orientations. However, this distribution is unknown. As a result, the calculated value of $37 \AA$ for the Förster distance is likely not correct, and it is not clear how much the performance of EGFP- $\Delta$ CAAX could be improved (or worsened) by manipulating the orientation of the transition dipole moment of GFP. Because $\kappa^{2}$ theoretically can range from 0 (orthogonal) to 4 (collinear), significant improvement may be possible.

Our attempt to force a change in relative orientation relied on Palmitoylation Site-EGFP- $\triangle$ CAAX [PS-EGFP- $\triangle$ CAAX, formerly known as dual-EGFP (Roorda et al., 2004)], which contains a C-terminal CAAX box identical to that of EGFP- $\triangle$ CAAX and, in addition, a palmitoylation site in an internal loop on the opposite end of the GFP $\beta$-barrel. Dual lipidation likely orients the transition dipole of PS-EGFP- $\triangle$ CAAX toward the membrane (Fig. $2 C$ ). As shown in Figure $2 D$, PS-EGFP- $\triangle$ CAAX exhibited a trend toward decreased $\Delta F / F$ compared with EGFP- $\Delta$ CAAX ( $p=0.15$; two-sided $t$ test). Because absolute fluorescence intensity was also reduced (data not shown), the decreased $\Delta F / F$ may reflect poor folding or chromophore maturation of PS-EGFP$\triangle \mathrm{CAAX}$ at $37^{\circ} \mathrm{C}$. Transition dipole orientation effects may be significant but difficult to isolate, because altering linker length alone has been shown to influence the distribution of dipole orientations (Windisch et al., 2006). It is therefore possible that transition dipole orientation effects play a role even in the increased voltage sensitivity of EGFP- $\triangle$ CAAX over EGFP-F.

How well do our experimental measurements fit the predictions of our model? In agreement with previous reports (Fernandez et al., 1983), measurements of charge translocation follow the Boltzmann distribution (Eq. 1) with values of $z$ ranging from -0.6 to -0.9 and $V_{0}$ ranging from -25 to $-35 \mathrm{mV}$ (Fig. $2 E$ ). To model experimental measurements of $\Delta F / F$, we first obtained values of $z$ and $V_{0}$ for each cell and then predicted relative quantum yield $Q$ using the full model (Eq. S7, available at www. jneurosci.org as supplemental material) with $y=30 \AA$ for EGFP-F and $y=10 \AA$ for all other constructs, and $R_{0}=42 \AA$ for CER- $\Delta$ CAAX and $R_{0}=37 \AA$ for all EGFP constructs. The agreement between model predictions of $Q$ and experimental measurements of $F$, which are related via Equation 3, was good (Fig. $2 F)$.

\section{Photon flux and two-photon shot noise of EGFP- $\triangle$ CAAX}

$\Delta F / F$ is not the only relevant parameter for assessing the performance of an optical voltage reporter (Sjulson and Miesenböck, 2007). The number of photons each cell can generate under realistic experimental conditions is equally important, because thick tissue imaging generally requires confocal or two-photon laser scanning microscopy for single-cell resolution, and measurements of fast events such as action potentials are often shot noise limited. To measure the photon flux generated by single cells expressing EGFP- $\triangle$ CAAX, two-photon voltage-clamp fluorometry was performed.

A representative cell shown in Figure 3 generates $\sim 7.5 \times 10^{3}$ photons/ms, with a $\Delta F / F$ of $\sim 16 \% / 100 \mathrm{mV}$ in $2 \mu \mathrm{M}$ DPA. If we approximate an action potential as a square pulse of $1 \mathrm{~ms}$ duration from -70 to $+30 \mathrm{mV}$, we can calculate the probability of detecting an action potential at a given false positive probability (Sjulson and Miesenböck, 2007). Choosing a false positive probability $P_{\mathrm{FP}}$ of $10^{-5} \mathrm{~ms}$, or one false positive action potential every $100 \mathrm{~s}$, the probability of detecting a $16 \%$ drop in amplitude lasting $1 \mathrm{~ms}$ is essentially $100 \%$.

\section{Optical imaging of electrical activity in vivo}

To test the ability of optimized hVOS to report electrical activity in vivo, we generated transgenic Drosophila expressing EGFP$\triangle$ CAAX and PS-EGFP- $\triangle$ CAAX under control of the UAS promoter (Brand and Perrimon, 1993). These flies were crossed with the OR83b-GAL4 line, which drives expression in $\sim 70 \%$ of all olfactory receptor neurons (ORNs) (Ng et al., 2002). Both EGFP constructs produced bright EGFP fluorescence in the antennal lobe that quenched after addition of DPA, consistent with membrane localization of the EGFP (data not shown). We then attempted two-photon imaging of ORN responses to odor stimulation in the antennal lobe but failed.

The failure to detect odor-evoked electrical activity in this setting could simply reflect the high temporal resolution of hVOS. Unlike synapto-pHluorin $(\mathrm{spH})$ or calcium indicators, the fluorescence responses of which decay over hundreds of milliseconds (Ng et al., 2002; Reiff et al., 2005), the response of hVOS terminates approximately three orders of magnitude faster, making imaging difficult. Because processes from $\sim 30$ individual ORNs are intertwined in each glomerulus at a subresolution scale, glomerular imaging necessarily records activity averaged across multiple neurons (Ng et al., 2002; Shang et al., 2007). If 

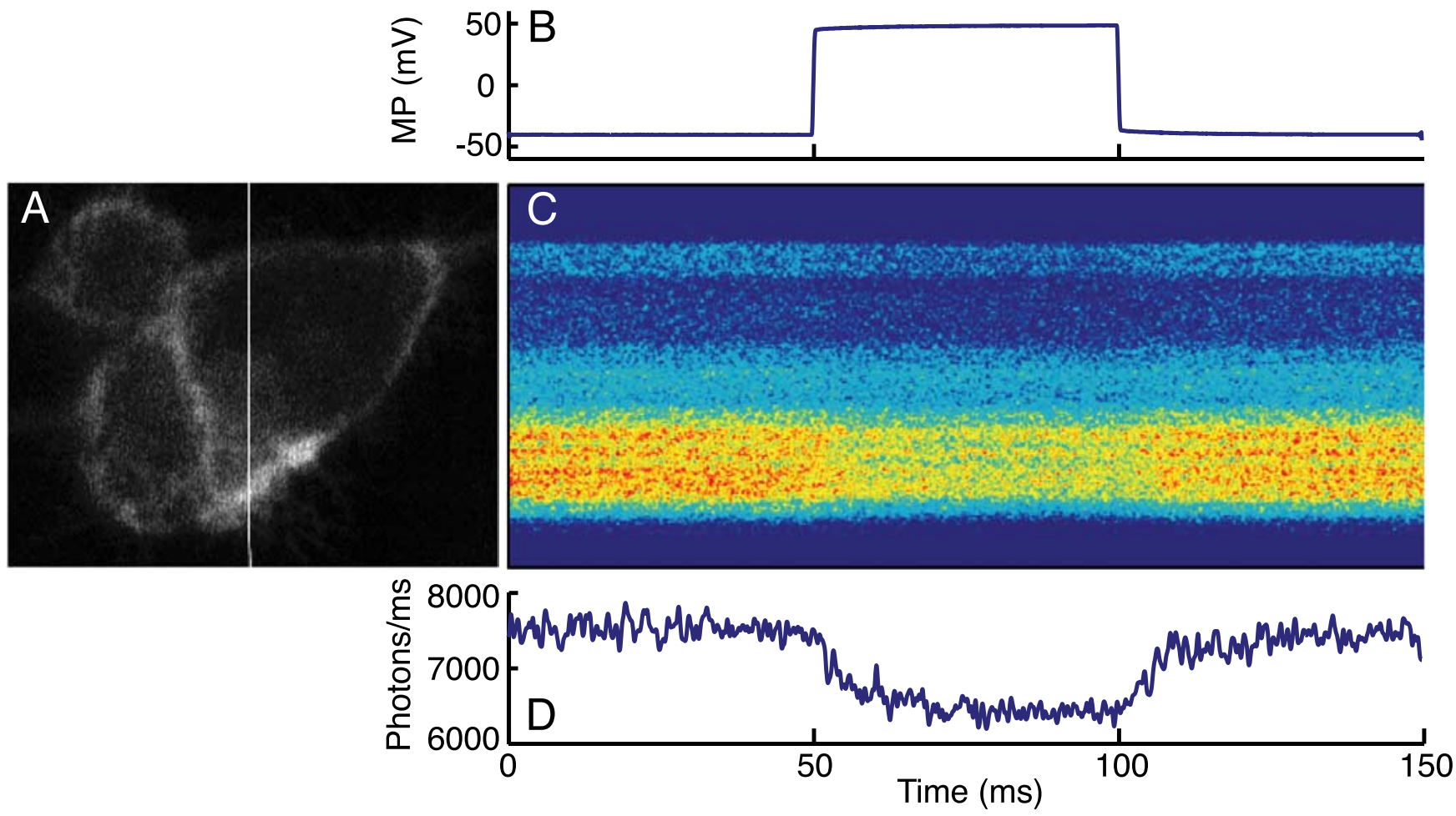

Figure 3. Two-photon voltage-clamp fluorometry yields a large signal with fast kinetics in $2 \mu \mathrm{m}$ DPA. A, Single focal plane image of HEK293 cells. The white line shows the location of the line scan. $\boldsymbol{B}$, Command potential for HEK293 cell in voltage-clamp experiment. $\boldsymbol{C}$, Single trial line scan data show decreased fluorescence at depolarized potentials. $\boldsymbol{D}$, Photon count estimate from line scan data. With photon counts in this range, shot noise would not be limiting for action potential recording. The slow on/off kinetics of the signal reflect a voltage-clamp artifact, because membrane capacitance was not compensated.
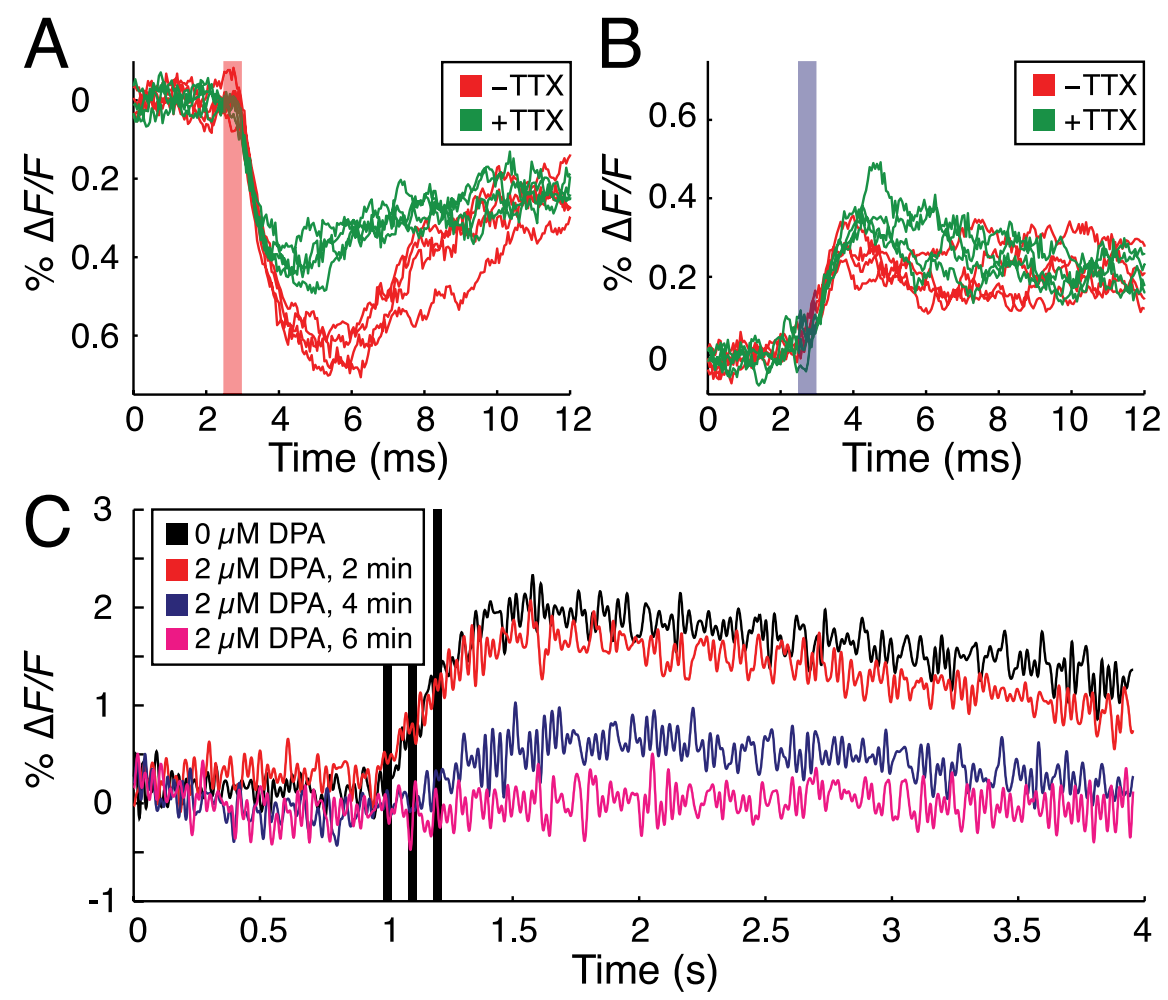

Figure 4. Imaging fast electrical activity in the Drosophila antennal lobe in vivo. A, Fluorescence responses to depolarizing current pulse (red bar) in $2 \mu \mathrm{m}$ DPA. Peak response amplitudes are significantly attenuated by $10 \mu \mathrm{m}$ tetrodotoxin (green trace; $p<0.01$ ). $\boldsymbol{B}$, Fluorescence responses to hyperpolarizing current pulse (blue bar) in $2 \mu \mathrm{m}$ DPA. Tetrodotoxin induces a small, but statistically significant, increase in response amplitude $(p<0.05)$. C, DPA $(2 \mu \mathrm{m})$ eliminates the spH response to electrical stimulation (black bars), consistent with inhibition of action potentials. each neuron were firing at $100 \mathrm{~Hz}$, and each action potential is $1 \mathrm{~ms}$ in duration, then each neuron would be maximally depolarized, at most, $10 \%$ of the time. Signal strength would be decreased by more than a factor of 10 , from $\sim 15$ to $\sim 1.5 \% \Delta F / F$, which is less than instrumentation noise.

To rectify this problem, we reasoned that activity could be imaged if action potentials were synchronized across ORNs. Accordingly, we combined wide-field fluorometry with direct electrical stimulation of the antennal nerve, which consists of ORN axons projecting to the antennal lobe, via a suction electrode. As shown in Figure $4 A$, voltage responses to depolarizing current pulses are detectable under these conditions. The response amplitudes, however, are $<1 \% \Delta F / F$, which is small compared with what would be expected if action potentials were initiated in every ORN axon. To test whether the optical signal was caused by action potentials or passive spread of depolarization, flies were tested in the presence and absence of $10 \mu \mathrm{M}$ tetrodotoxin (TTX), a voltage-gated sodium channel blocker. Although a significant passive response remained in the presence of TTX, the peak amplitude of the response to depolarizing pulses was significantly decreased in all flies tested $(p<0.01$ in each fly; two-sided $t$ test). The response to hy- 
perpolarizing pulses interleaved with the depolarizing pulses was increased slightly $(p<0.05)$, as would be expected if TTX increased input resistance (Fig. $4 B$ ). Consistent with this interpretation, the response amplitudes of both depolarizing and hyperpolarizing pulses remained constant over the duration of control experiments performed in the absence of TTX ( $p=0.30$; twosided $t$ test).

Although the responses are clearly TTX responsive, they are inconsistent with synchronous action potential generation in a large fraction of ORN axons: the $\Delta F / F$ of the response is $<10 \%$ of that expected for synchronized, full-scale action potentials, and the signal outlasts the duration of a single spike by several ms. Together, these characteristics suggest that antennal nerve stimulation caused subthreshold depolarizations rather than propagating action potentials in ORNs. The likely factor limiting action potential initiation or propagation is the increased capacitive load caused by the presence of $2 \mu \mathrm{M}$ DPA. To test this notion, we performed wide-field fluorometry experiments with suction electrode stimulation of the antennal nerve, but using $\mathrm{spH}$ rather than the optimized hVOS as a read-out of ORN activity (Miesenböck et al., 1998; Ng et al., 2002; Shang et al., 2007). In the absence of DPA, spH responses were readily evoked (Fig. 4C, black line). After addition of $2 \mu \mathrm{M}$ DPA to the bath, however, these responses gradually faded as the density of DPA in axonal membranes increased (Fig. $4 C$, colored lines). In the steady state, which was reached after several minutes in $2 \mu \mathrm{M}$ DPA, all electrical excitability was suppressed. Because the off-rate of DPA is so low that DPA incorporation into membranes is practically irreversible (Fernandez et al., 1983; DiFranco et al., 2007), removal of DPA from the extracellular solution did not restore $\mathrm{spH}$ signals.

To determine whether DPA concentrations below $2 \mu \mathrm{M}$ could yield a sufficient $\Delta F / F$ without inhibiting action potentials, we repeated the suction electrode stimulation experiments at several lower DPA concentrations. Concentrations below $750 \mathrm{~nm}$ DPA did not yield detectable signals, whereas signals recorded at 750 nM and $1 \mu \mathrm{M}$ DPA exhibited amplitudes smaller than $0.5 \%$ and waveforms consistent with subthreshold depolarizations (data not shown).

\section{Model predictions for further optimization of optical voltage reporters}

To examine the plausibility of future strategies for optimization of optical voltage reporters, we first determined theoretical upper bounds for $\Delta Q$ and $\Delta Q / Q$ and compared them to predicted values for hVOS and other candidate voltage reporters. We then applied signal detection theory in a model of realistic photon shot noise to determine how these values translate to performance in optical detection of action potentials.

Theoretical limits on $\Delta Q$ and $\Delta Q / Q$ are based on constraints that are common to all genetically encoded optical voltage reporters. The first, and most fundamental, of these is thermal noise, which limits the voltage sensitivity of mobile probe charges, as described by the Boltzmann distribution (Eq. 1). For a DPA molecule, where $|z|=0.6-1$ (Fernandez et al., 1983; Chanda et al., 2005a; DiFranco et al., 2007), the two voltage-dependent states of Equation 1 correspond to the molecule inhabiting the inner or outer membrane leaflet. At $25^{\circ} \mathrm{C}$, a $100 \mathrm{mV}$ change in membrane potential cannot drive all DPA molecules across the membrane; rather, at most, 53\% $(|z|=$ 0.6) (Fig. 5C, solid black line) to 75\% $(|z|=1)$ (Fig. 5C, dotted black line) of all DPA molecules will translocate. Mobile probes with more than one elementary charge, such as gating charges in voltage-gated ion channels, have higher voltage sensitivity: with $|z|=4$, over $99.9 \%$ of molecules switch states per $100 \mathrm{mV}$, whereas $|z|=13$, the approximate value for the Shaker channel (Aggarwal and MacKinnon, 1996) leads to near-total charge translocation over $100 \mathrm{mV}$ (Fig. 5C, dashed black lines).

The second element of our analysis is the efficiency of coupling between voltage-dependent state and fluorescence. The theoretically optimal voltage-sensitive fluorophore has perfect coupling between voltage-dependent state and fluorescence; in other words, there is full fluorescence in one voltage-dependent state and zero fluorescence in the other. Under these conditions, the relative quantum yield $Q$ is the same as the probability of inhabiting the outer leaflet (Fig. $5 C$, black lines), with a maximum $\Delta Q$ per $100 \mathrm{mV}$ ranging from 0.53 for $|z|=0.6$ to 0.75 for $|z|$ $=1$ (Fig. $5 D$, black bars); this results in a $\Delta Q / Q$ per $100 \mathrm{mV}$ ranging from $0.69(|z|=0.6)$ to $0.86(|z|=1)$ at maximal $\Delta Q$ (Fig. $5 D$, black bars) (for a discussion of maximizing $\Delta Q / Q$, see supplemental material, available at www.jneurosci.org). There are parameter regimes in which these theoretical limits do not apply to the hVOS model, for example, at extremely high DPA densities where each GFP interacts with multiple DPA molecules. At physiologically tolerated DPA densities, however, the approximation is adequate.

In addition to being smaller than the theoretical limit, the maximal $\Delta Q$ for hVOS also depends on the density of FRET acceptors in the membrane. As a starting point, we therefore examined the maximum $\Delta Q$ per $100 \mathrm{mV}$ when membrane capacitance is increased by $0.2 \mu \mathrm{F} / \mathrm{cm}^{2}$, or $\sim 20 \%$, which corresponds to a range from $\sim 1300$ molecules $/ \mu \mathrm{m}^{2}(|z|=1)$ to $\sim 2200$ molecules $/ \mu \mathrm{m}^{2}(|z|=0.6)$ (for details, see supplemental material, available at www.jneurosci.org as supplemental material). Using the full model of hVOS discussed above, we first analyzed the impact of varying the Förster distance $R_{0}$ and GFP-membrane distance $y$ over wide ranges with $|z|=1$ (Fig. $5 A$ ). Consistent with our experimental findings, $\Delta Q$ is larger at shorter GFP-membrane distances when $R_{0}=37 \AA$. But, surprisingly, the model predicts, in the (hypothetical) regime of large Förster distances (i.e., for $R_{0}>\sim 50 \AA$ ), an increase in $\Delta Q$ as the GFP moves further from the membrane (Fig. $5 A$ ). At first sight, this result seems counterintuitive, because the fractional change in distance resulting from DPA translocation is reduced as the GFP-membrane distance increases. However, this reduction is offset by decreased quenching from DPA molecules in the outer leaflet. Compared with the predicted $\Delta Q$ of the DPA/EGFP- $\Delta$ CAAX pair $\left(R_{0}=37 \AA ; y=10 \AA\right)$, increasing $R_{0}$ and $y$ in the optimal "long-range" hVOS analyzed in our model $\left(R_{0}=100 \AA ; y=50 \AA\right)$ more than doubles the maximum $\Delta Q$ per $100 \mathrm{mV}$ from 0.03 to 0.08 (Fig. $5 A, D$, red and green crosses), representing 4 and $10 \%$ of the theoretical limit, respectively. Unfortunately, experimental avenues for increasing the Förster distance into the long-range regime are not immediately apparent, at least for the DPA/EGFP pair studied here. Repeating these calculations for $|z|=0.6$ yields surprisingly similar results (Fig. $5 C, D$ ), suggesting that characteristics of hVOS are relatively stable across the physiologic range of $|z|$, with increased DPA surface density and quenching efficiency offsetting the decreased voltage sensitivity resulting from decreasing $|z|$.

A second potential strategy for improving the performance of hVOS is the "fluorescent acceptor" configuration, in which fluorescent hydrophobic anions are used instead of DPA (Gonzalez and Tsien, 1995, 1997; Cacciatore et al., 1999; Tsien and Gonza- 

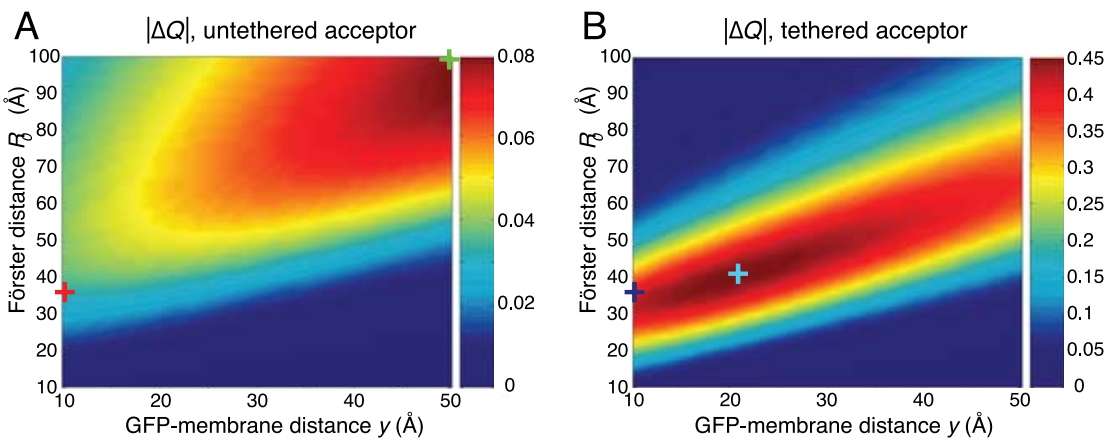

C
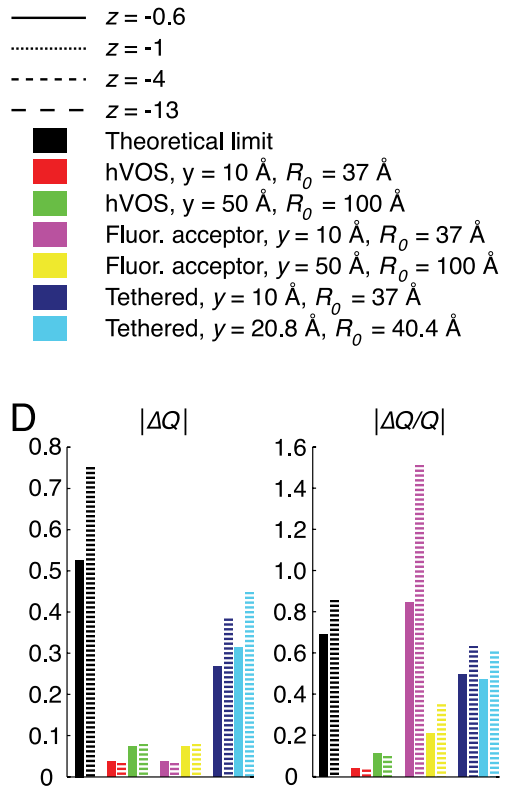

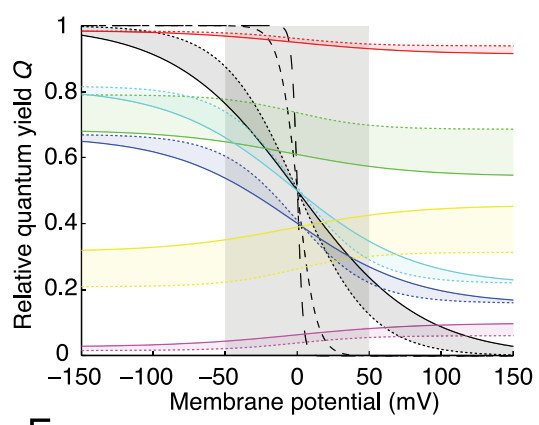

$\mathrm{E}$

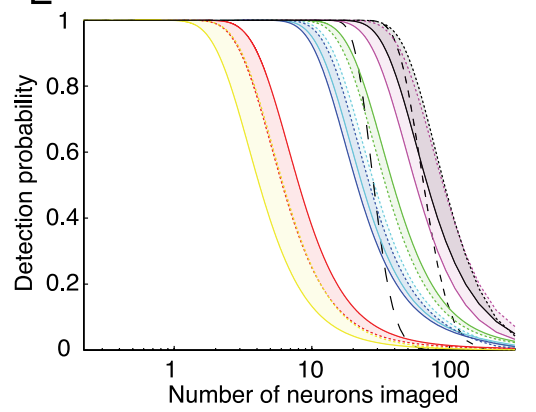

Figure 5. Theoretical performance limits and future optimization of hVOS. $A$, Predicted $|\Delta Q|$ per $100 \mathrm{mV}$ for hVOS at an acceptor density of 1300 molecules $/ \mu \mathrm{m}^{2},|z|=1$. The DPA/EGFP- $\Delta$ CAAX pair $\left(y=10 \AA ; R_{0}=37 \AA\right.$ ) yields a $|\Delta Q|$ of 0.03 (red cross), whereas the optimal values tested ( $y=50 \AA ; R_{0}=100 \AA$ ) yield a $|\Delta Q|$ of 0.08 (green cross). Note that low values of $y$ are optimal for realistic values of $R_{0}$, whereas large $y$ is optimal for very large values of $R_{0}$. B. Predicted $|\Delta Q|$ per $100 \mathrm{mV}$ for the tethered acceptor configuration, in which the FRET acceptor is covalently linked to the GFP, at a density of 1300 molecules $/ \mu \mathrm{m}^{2}$, $|z|=1$. For tethered-DPA/EGFP- $\Delta$ CAAX ( $\left.y=10 \AA ; R_{0}=37 \AA\right)$, the $|\Delta Q|$ is 0.38 (blue cross), whereas the optimal values tested ( $y=20.8 \AA ; R_{0}=40.4 \AA$ ) yield a $|\Delta Q|$ of 0.48 (cyan cross). Note that optimal $|\Delta Q|$ is attained near experimentally realistic values of $y$ and $R_{0}$. C, Relative quantum yield $Q$, as a function of $V_{m}-V_{0}$, at a maximum induced capacitance of $0.2 \mu \mathrm{F} / \mathrm{cm}^{2}$. The shaded gray box denotes the $100 \mathrm{mV}$ window producing maximum $|\Delta Q|$. The theoretical limit of $|\Delta Q|$ increases significantly with increasing $|z|$ (black lines). Values of $Q$ calculated at $|z|=0.6$ (solid line) and $|z|=1$ (dotted line) represent bounds on optimal values of $Q$ for monovalent anions such as DPA, whereas larger values of $|z|$ (dashed lines) represent upper bounds on $|\Delta Q|$ for reporters with cooperative charge movement, such as GFP-ion channel fusions. The ideal hVOS ( $y=50 \AA ; R_{0}=100 \AA$ ) yields a modestly increased $|\Delta Q|$ (green vs red), whereas the tethered acceptor configuration produces much larger increases in $|\Delta Q|$ (blue and cyan). The use of fluorescent FRET acceptor molecules does not change $|\Delta Q|$ (magenta and yellow). $\boldsymbol{D},|\Delta Q|$ and $|\Delta Q / Q|$ for the three configurations are compared with theoretical limits with $|z|=0.6$ (solid black bars) or $|z|=1$ (dashed black bars). The tethered acceptor configuration (blue and cyan bars) produces much larger $|\Delta Q|$ and $|\Delta Q / Q|$ than $\mathrm{hVOS}$ (green and red bars), whereas the fluorescent acceptor configuration (magenta and yellow bars) produces identical $|\Delta Q|$ with much larger $|\Delta Q / Q|$. The $|\Delta Q / Q|$ for the fluorescent acceptor model is able to surpass the theoretical limit, because its fluorescence increases, rather than decreases, with depolarization (see Results for discussion). E, Probability of action potential detection as a function of number of neurons imaged. Performance of the theoretically optimal voltage reporter improves as $|z|$ increases from 0.6 (solid black line) to 1 (dotted black line) and then deteriorates as $|z|$ increases further (dashed black lines). Despite substantially lower $|\Delta Q|$ and $|\Delta Q / Q|$, the ideal hVOS $\left(y=50 \AA ; R_{0}=100 \AA\right)$ has better performance than the tethered acceptor model (green vs blue/cyan), caused by greater density of fluorophores, whereas the fluorescent acceptor model ( $y=10 \AA ; R_{0}=37 \AA$ ) performs near the theoretical limit (magenta vs black).

lez, 2002; DiFranco et al., 2007), and the fluorescence signal is recorded from the acceptor instead of the donor. In analogy to $Q$, the relative quantum yield of the donor, we defined a quantity $Q_{A}$ as follows:

$$
Q_{A}=\frac{\phi_{a}(1-Q)}{\phi_{d}}
$$

where $\phi_{a}$ and $\phi_{d}$ are the fluorescence quantum yields of the acceptor and donor, respectively. $Q_{A}$ represents the ratio of two probabilities: the probability of donor excitation leading to photon emission by an acceptor divided by the probability of that donor emitting a photon if no acceptors were present. Assuming $\phi_{a}$ and $\phi_{d}$ to be equal, we used the full hVOS model to calculate $Q$, with $\Delta Q_{A}$ equal to $-\Delta Q$ (Fig. $5 A, D)$. Although the amplitudes of $\Delta Q_{A}$ and $\Delta Q$ are the same, $Q_{A}$ is significantly decreased relative to $Q$, substantially increasing $\Delta Q_{A} / Q_{A}$. With parameters of $y=$ $10 \AA$ and $R_{0}=37 \AA$, the $\Delta Q_{A} / Q_{A}$ is increased to a range of $0.85-1.51$ from a $\Delta Q / Q$ of $0.03-0.04$ (Fig. $5 D$, magenta vs red bars). The acceptor fluorescence signal is capable of exceeding the theoretical $\Delta Q / Q$ limit of 0.86 , because this limit holds only when fluorescence decreases with depolarization. Because acceptor fluorescence increases with depolarization, the thermal noise-limited $\Delta Q_{A} / Q_{A}$ for a 100 $\mathrm{mV}$ depolarizing pulse at maximal $\Delta Q_{A}$ is 6.0; the large $\Delta Q_{A} / Q_{A}$ of $1.51(|z|=1)$ attains $\sim 25 \%$ of this theoretical maximum (supplemental material, available at www. jneurosci.org). Consistent with these predictions, DiFranco et al. (2007) recently reported that combining EGFP-F with $\mathrm{DiBAC}_{4}(5)$, a fluorescent dye, rather than $D P A$, leads to a significant increase in $\Delta F / F$. Although $\operatorname{DiBAC}_{4}(5)$ translocation is likely too slow to record neuronal action potentials, it may be sufficient for following slower phenomena such as muscle action potentials or rhythmic subthreshold activity.

Another benefit of fluorescent acceptor molecules is that a potentially significant fraction of any membrane-targeted GFP will be either cytoplasmic or associated with intracellular membranes, thereby contributing to background fluorescence. With fluorescent acceptors, only GFP molecules in close proximity to the acceptors (i.e., at the plasma membrane) contribute to the signal. The downsides of fluorescent acceptors include increased phototoxicity resulting from fluorescence excitation within the membrane bilayer and, for all fluorescent dyes reported to date (Cacciatore et al., 1999), membrane translocation speeds much slower than DPA.

A third potential strategy for increasing $\Delta Q$ is the "tethered acceptor" configuration, in which the local DPA concentration at each GFP is increased by covalently linking the FRET acceptor molecule to the GFP via a flexible "leash," the length of which exceeds the thick- 
ness of the membrane. Several technologies for selective covalent attachment of exogenous molecules to specific proteins have been demonstrated (Griffin et al., 1998; Chen et al., 2005; Marks and Nolan, 2006), suggesting that this approach is feasible, at least in principle, although covalent association with the linker would likely slow translocation of the FRET acceptor. It is important to stress that the tethered acceptor model represents a special case of the general class of optical voltage reporters in which the fluorophore and mobile charges are physically associated in a single complex; this class also includes GFP-channel fusions. The tethered acceptor configuration represents the most direct analog of hVOS incorporating physical association; to evaluate the effects of this association, we extended our model to include a tethered acceptor using mathematical methods adapted from the study by Wolber and Hudson (1979) (supplemental Methods, available at www.jneurosci.org as supplemental material) and estimated the maximum $\Delta Q$ per $100 \mathrm{mV}$ (Fig. $5 B$ ). The maximum $\Delta Q$ for the tethered-DPA/EGFP- $\Delta$ CAAX pair $\left(R_{0}=37 \AA ; y=10\right.$ $\AA$ ) is increased to a range of $0.27-0.38$, representing $\sim 51 \%$ of the theoretical limit; this is $\sim 10$-fold greater than the untethered hVOS DPA/EGFP- $\Delta$ CAAX pair (Fig. $5 D$, blue vs red bars). The parameters yielding the largest $\Delta Q$ per $100 \mathrm{mV}$ are $R_{0}=40.4 \AA$, $y=20.8 \AA,|z|=1: \Delta Q$ then increases to $0.31-0.45$, or $\sim 60 \%$ of the theoretical limit (Fig. $5 D$, cyan bar). $\Delta Q / Q$ is similarly increased relative to optimized hVOS, but less so than for the fluorescent acceptor configuration (Fig. 5D).

\section{Implications for optical detection of action potentials}

Because the relationship between $\Delta Q, \Delta Q / Q$, and the ability to detect action potentials is complex, we calculated the effects of photon shot noise on the probability of action potential detection using realistic experimental values for excitation power and collection efficiency in high-speed two-photon microscopy. We modeled an action potential as a $100 \mathrm{mV}$ step of $1 \mathrm{~ms}$ duration and, approximating Poisson photon statistics using the incomplete Gamma function, calculated the detection probability using the Neyman-Pearson criterion (Whalen, 1971) with a false detection probability of $10^{-3} / \mathrm{ms}$, or one false spike per second, a less stringent criterion than used for our previous signal detection calculation (Fig. 3). A key factor in this analysis is the tolerated density of fluorophores at the membrane, which in turn depends on $|z|$, the number of mobile probe charges associated with each fluorophore. For example, a maximum tolerated membrane capacitance increase of $\sim 20 \%\left(0.2 \mu \mathrm{F} / \mathrm{cm}^{2}\right)$ corresponds to $\sim 2200$ fluorophores $/ \mu \mathrm{m}^{2}$ when $|z|=0.6$, or $\sim 1300$ fluorophores $/ \mu \mathrm{m}^{2}$ for $|z|=1$. Because the maximum induced capacitance is linearly proportional to $|z|$ (supplemental material, available at www. jneurosci.org), additional increases in $|z|$ require proportional decreases in fluorophore density to maintain the same additional capacitive load. For example, $|z| \approx 13$ for most fusions of GFP with voltage-gated cation channels (Aggarwal and MacKinnon, 1996), meaning that the sensor surface density would have to be further decreased 13 -fold to $\sim 100$ molecules $/ \mu \mathrm{m}^{2}$ to maintain a $20 \%$ capacitance increase. Therefore, increasing values of $|z|$ require a tradeoff between decreased fluorophore density and increased voltage sensitivity.

We first examined the performance of the ideal voltagesensitive fluorophore, that is, a fluorophore switched completely on and off by changes in voltage-dependent state, at densities causing a capacitance increase of $20 \%$. Under typical imaging conditions $(\lambda=910 \mathrm{~nm}$; NA $=0.8)$, with $|z|$ ranging from 0.6 to 1 , the number of fluorophore molecules inside the two-photon excitation volume ranges from $\sim 68(|z|=1)$ to $\sim 115(|z|=0.6)$
(Zipfel et al., 2003). Assuming pulsed excitation at a rate of 82 $\mathrm{MHz}$, a pulse intensity capable of exciting $1 \%$ of all fluorophores in the excitation volume per pulse (a realistic value when limited by photobleaching), a quantum yield of 0.6 , and a collection efficiency of $2 \%, \sim 660-1100$ photons/ms will be collected. It is therefore possible to collect $\sim 660-1100$ photons/ms from one cell, $\sim 330-550$ photons/ms per cell from two cells, and so forth, depending on the value of $|z|$ in the range of $0.6-1$. We thus plotted the probability of detecting an action potential as a function of the number of neurons sampled, noting that the differences between various values of $|z|$ reflect a tradeoff between more photons collected at lower $|z|$ and higher voltage sensitivity at higher $|z|$. Interestingly, the number of neurons that can be imaged is largest at $|z|=1$ and decreases as $|z|$ decreases to 0.6 (Fig. $5 E$, black interval); calculations performed at intermediate values of $|z|$ indicate that this decrease is monotonic (data not shown), suggesting that the positive effect of increasing voltage sensitivity is dominant in the regime of $|z|<1$. However, increasing $|z|$ to 2 leads to performance nearly identical to $|z|=1$, whereas additional increases of $|z|$ to values of 4 or 13 , in the range of voltagegated cation channels, lead to significant decreases in performance (Fig. 5E, dashed black lines). The negative effects of decreasing photon counts thus dominate in the range of $|z|>2$.

We next examined the performance of nonideal fluorophores, which yield a smaller $\Delta Q$ than the theoretical limit. The simplest of these is the tethered model, which represents the general class of reporters in which the fluorophore is physically associated with mobile probe charges. This class includes both the tethered acceptor configuration and GFP fusions with voltage-gated cation channels (Siegel and Isacoff, 1997; Sakai et al., 2001; Ataka and Pieribone, 2002; Guerrero et al., 2002). The tethered-DPA/ EGFP- $\Delta$ CAAX pair ( $y=10 \AA ; R_{0}=37 \AA$ ) and optimal tethered pair ( $y=20.8 \AA ; R_{0}=40.4 \AA$ ) yield a $\Delta Q$ of 51 and $60 \%$ of the theoretical limit, respectively (Fig. $5 D$, blue and cyan bars). Although this $\Delta Q$ amounts to more than half of the theoretical limit in both cases, the number of neurons that can be recorded with a detection probability of $50 \%$ for the tethered-DPA/EGFP$\triangle$ CAAX pair is only $29-31 \%$ of the number in the ideal case (Fig. $5 E$, blue vs black), or $31-34 \%$ of the ideal for the optimal tethered pair (Fig. 5E, cyan vs black). It is difficult to make precise predictions for GFP fusions with voltage-gated cation channels, because the mechanisms of fluorescence modulation are generally unclear. However, performance is likely to be poor given that the $\Delta F / F$ of all existing channel-based probes is at least one order of magnitude lower than the predicted $\Delta Q / Q$ of the tethered acceptor configuration (Siegel and Isacoff, 1997; Sakai et al., 2001; Ataka and Pieribone, 2002; Guerrero et al., 2002). This problem is compounded by the large number of gating charges associated with each fluorophore (Aggarwal and MacKinnon, 1996), which compromises performance even in the ideal case (Fig. $5 E$, dashed black lines). In practice, however, the performance of these probes may be improved by attaching several GFP molecules per channel tetramer or by exploiting the hysteretic dynamics of channel inactivation to generate fluorescence responses that outlast the duration of the action potential itself (Siegel and Isacoff, 1997). Neither of these strategies are considered in our analysis.

The analogous calculation for hVOS and the fluorescent acceptor configuration is more complex, because the ratio of fluorophores to probe charges is not fixed. For hVOS, if the inner membrane leaflet was filled to capacity with GFP molecules, there would be $\sim 10^{5} \mathrm{GFP}$ molecules/ $\mu \mathrm{m}^{2}$, compared with $1300-2200$ DPA molecules $/ \mu \mathrm{m}^{2}$. If $1 \%$ of these GFP molecules is excited by each laser pulse, there are $\sim 1000$ excited GFP molecules $/ \mu \mathrm{m}^{2}$, 
rather than 13-22 in the tethered acceptor configuration. As a result, small changes in the signal are much easier to detect; despite exhibiting a $\Delta Q$ and $\Delta Q / Q$ several-fold lower than the tethered acceptor configuration (Fig. $5 D$ ), the performance of the ideal hVOS $\left(R_{0}=100 \AA ; y=50 \AA\right)$ is better than that of the optimal tethered acceptor configuration (Fig. $5 E$, green vs cyan). Using realistic parameters for optimized hVOS $\left(R_{0}=37 \AA ; y=\right.$ $10 \AA$ ), however, enables observation of approximately fivefold fewer neurons than in the tethered acceptor configuration (Fig. $5 E$, red vs blue).

To evaluate the performance of the fluorescent acceptor configuration, we applied the additional constraint that the excitation probabilities of both the donor and acceptor are $<1 \%$ with each laser pulse. Limiting excitation to low levels is particularly important with small molecule fluorophores residing in the membrane, because both photobleaching and phototoxicity are more severe than with membrane-peripheral GFP. The proportion of acceptors excited with each pulse is determined by the following equation:

$$
p_{\mathrm{ex}_{A}}=\frac{D_{T}}{A_{T}} p_{\mathrm{ex}_{D}}(1-Q),
$$

where $A_{T}$ and $D_{T}$ represent the membrane density of acceptor and donor, respectively, whereas $p_{\mathrm{ex}_{A}}$ and $p_{\mathrm{ex}_{D}}$ represent excitation probabilities. For realistic values of $A_{T}, D_{T}$, and $Q, p_{\mathrm{ex}_{A}}$ is several-fold larger than $p_{\operatorname{ex}_{D}}$, requiring that illumination intensity be decreased for $p_{\mathrm{ex}_{4}}$ to equal 0.01 in the resting membrane potential state. The fluorescent FRET acceptor configuration is therefore unable to take full advantage of the higher photon counts associated with hVOS; however, the much smaller background fluorescence at resting potentials more than compensates for this, producing performance nearly identical to the theoretical limit (Fig. 5E, magenta vs black).

\section{Discussion}

\section{Increased membrane capacitance as fundamental limitation}

Increased membrane capacitance is a significant underappreciated drawback of genetically encoded optical voltage reporters (Chanda et al., 2005b; Sjulson and Miesenböck, 2007). By using a rational model-based approach, we were able to optimize hVOS for lower DPA concentrations, thus enabling recordings of electrical activity in vivo. Nevertheless, increased membrane capacitance remains a limiting factor: our results demonstrate that even low concentrations of DPA in the bath are more poorly tolerated than previous studies suggest (Chanda et al., 2005b).

This apparent discrepancy is likely caused by large differences in the effective DPA concentrations in different preparations; these differences are only poorly reflected in the nominal DPA concentrations in the bath. In addition, different methods are used to elicit and detect action potentials, making direct comparisons difficult. For instance, Chanda et al. (2005b) reported that cultured neurons fire action potentials in $3 \mu \mathrm{M}$ DPA, but this result was obtained by measurement at the site of current injection. The absence of detectable $\mathrm{spH}$ responses at ORN terminals, which lie at considerable distance from the site of antennal nerve stimulation, may thus reflect a failure of action potential propagation rather than initiation. Chanda et al. (2005b) also reported that population spikes in the hippocampal CA1 region have a normal waveform in $4 \mu \mathrm{M}$ DPA; however, their preparation was a $350-\mu \mathrm{m}$-thick tissue slice containing a significant amount of myelin, which is expected to act as a sink for hydrophobic DPA molecules and/or slow their penetration into the slice. The fly brain, in contrast, contains no myelin and is only $\sim 250 \mu \mathrm{m}$ wide, with the antennal nerve lying entirely superficial. As a result, the effective concentration of DPA at the membranes of neurons in the hippocampal slice is likely much lower than in the fly antennal nerve, although the bath concentration is higher.

It is worth noting that it has not been conclusively demonstrated that the deleterious effects of DPA are mediated solely by increased membrane capacitance. Adsorption and desorption of DPA carries some current across the membrane and would be expected to influence membrane potential, although Chanda et al. (2005b) estimate the effect to be $<2 \mathrm{mV}$ at $5 \mu \mathrm{M} \mathrm{DPA}$, consistent with the extremely low off rate of DPA (Fernandez et al., 1983; DiFranco et al., 2007). DPA is also protonatable, meaning that it can act as a protonophore with some ability to deplete mitochondrial $\mathrm{pH}$ gradients, but this effect is likely negligible at the concentrations used here, because of the low $\mathrm{pK}_{\mathrm{a}}$ of 2.66 (Gaboriaud, 1966). Finally, it is possible that DPA exhibits additional unrelated pharmacologic effects that are more severe in flies. These issues could be addressed by future studies examining the effects of other hydrophobic anions, such as tetraphenylborate (Szabo, 1977), on excitability.

More importantly, any source of increased membrane capacitance will necessarily influence important physiological parameters, and the mere fact that action potentials are not inhibited does not guarantee the presence of normal physiological function. For example, it is well known that membrane capacitance is a primary factor limiting axonal propagation speed, and mammalian brain evolution has devoted significant resources to minimizing conduction delays within the brain, including both myelination and scaling relationships dictating disproportionately large axonal diameters with increasing brain size (Harrison et al., 2002). Additionally, increasing membrane capacitance increases the membrane time constant proportionally, and the relationship between the membrane time constant and mean interspike interval is what determines whether a neuron acts as an "integrator" or "coincidence detector" (König et al., 1996). Intact neural circuits encode information and exhibit correlated activity on a timescale of 10-30 ms (Victor and Purpura, 1996; Harris et al., 2003), which matches the membrane time constant, presumed EPSP width (Spruston and Johnston, 1992), and period of the gamma oscillation, leading some authors to speculate that the membrane time constant and EPSP width are the rate-limiting factors in cognitive processing (Harris, 2005). Future studies are needed to explore the effects of increasing conduction delays and membrane time constants, however subtly; in our view, it would be optimistic to assume that large increases would be well tolerated.

\section{Capacitive silencing of neuronal activity}

On the flip side, our demonstration that even modest capacitance increases inhibit action potential generation and/or propagation suggests a new general strategy for reversible genetically targeted silencing of neuronal activity. Although most existing approaches rely on the cell type-specific expression of potassium conductances that clamp the neuronal membrane potential at hyperpolarized levels (Baines et al., 2001; Nitabach et al., 2002), these manipulations can alter intracellular ion concentrations, leading to cell death (Nadeau et al., 2000). The expression of nonconducting channels (Perozo et al., 1993) or isolated voltage-sensing modules (Murata et al., 2005) that suppress excitation by virtue of their gating charges promises to circumvent this problem. However, it is possible that increasing membrane capacitance on gene regulation timescales would enable homeostatic upregula- 
tion of endogenous conductances to partially offset the silencing effect.

\section{Imaging technology as a limiting factor}

Imaging fast events such as action potentials in intact tissue is challenging for two reasons (Sjulson and Miesenböck, 2007). First, single-cell resolution in thick, scattering tissue typically requires laser scanning microscopy. Because the laser is scanned across the sample, it is technically difficult to achieve sufficiently high sampling rates, although progress has recently been made with optical random access in two (Iyer et al., 2006; Salome et al., 2006) and three (Reddy and Saggau, 2005) dimensions. Depthresolved wide-field multiphoton microscopy may also become a reality (Oron et al., 2005).

A more serious limitation of laser scanning microscopy is that the number of photons collected is low, further compounding the capacitance-limited low membrane density of fluorophores. Fortunately, collection efficiency can be improved significantly through the use of high NA objectives, mirror-based objectives (Vucinic et al., 2006), or collection of light through the condenser (Mainen et al., 1999; Roorda et al., 2004). The use of fluorophores with higher two-photon cross sections, such as quantum dots $(\mathrm{Pu}$ et al., 2006), would also significantly improve the situation. The results in Figure $5 E$ are not fundamental but based on collection efficiency and fluorophore characteristics: increases in imaging efficiency will proportionally increase the number of neurons that can be imaged. Likewise, using potentiometric dyes that do not increase membrane capacitance enables higher fluorophore membrane densities and signal-to-noise ratios (Fisher et al., 2008). Nevertheless, imaging technology currently remains a significant limiting factor in detecting action potentials and other fast electrical activity in the intact brain.

\section{Limitations of our modeling approach}

Our models make two major simplifications: first, we ignore orientational effects of the molecules, including the fact that relative orientation, and therefore $R_{0}$, varies as a function of lateral distance along the membrane. However, one modeling study has shown that this may not be too serious an issue, because orientational effects are generally small in FRET between planes of a bilayer (Domanov and Gorbenko, 2002). A more significant assumption is that the GFP and DPA molecules are uniformly distributed in the membrane. This is almost certainly not true; biological membranes possess significant lateral heterogeneity, and both DPA molecules and GFP prenyl anchors likely partition preferentially into certain sections of the membrane (Zacharias et al., 2002). Our experimental measurements of $\Delta F / F$ also necessarily include contributions from GFP not localized to the plasma membrane. The application of total internal reflection fluorescence microscopy in future studies could allow selective measurement of plasma membrane fluorescence (Thompson et al., 1981), enabling direct experimental measurement of relative quantum yield $Q$. Despite these qualifications, our model produced predictions that allowed us to optimize hVOS and image fast electrical activity in the fly olfactory system. In addition, it has generated testable predictions regarding possible strategies for the future improvement of hVOS.

Our signal detection analysis in Figure 5 addresses only the specific problem of multisite recording of action potentials from the intact brain. This is not necessarily the only, or even the most important, potential application of genetically encoded optical voltage reporters. For example, the ability to target expression to specific cell classes may provide a powerful tool for studying the origins, mechanisms, and modulation of rhythmic activity in the cortex of intact animals, not unlike a local field potential recording, but with genetic resolution. Additionally, FRET acceptor dyes can be chosen with slower translocation time constants that match the time scales of the phenomena being studied, effectively reducing the capacitive load for fast electrical activity. Alternatively, the low phototoxicity relative to voltage-sensitive dyes may be advantageous in studies of dendritic integration where averaging across many trials is possible. These represent areas in which this or other optimized versions of hVOS are likely to make significant contributions in the near future.

\section{References}

Aggarwal SK, MacKinnon R (1996) Contribution of the S4 segment to gating charge in the Shaker K+ channel. Neuron 16:1169-1177.

Ataka K, Pieribone VA (2002) A genetically targetable fluorescent probe of channel gating with rapid kinetics. Biophys J 82:509-516.

Baines RA, Uhler JP, Thompson A, Sweeney ST, Bate M (2001) Altered electrical properties in Drosophila neurons developing without synaptic transmission. J Neurosci 21:1523-1531.

Brand AH, Perrimon N (1993) Targeted gene expression as a means of altering cell fates and generating dominant phenotypes. Development 118:401-415.

Buzsaki G (2004) Large-scale recording of neuronal ensembles. Nat Neurosci 7:446-451.

Cacciatore TW, Brodfuehrer PD, Gonzalez JE, Jiang T, Adams SR, Tsien RY, Kristan Jr WB, Kleinfeld D (1999) Identification of neural circuits by imaging coherent electrical activity with FRET-based dyes. Neuron 23:449-459.

Chanda B, Asamoah OK, Blunck R, Roux B, Bezanilla F (2005a) Gating charge displacement in voltage-gated ion channels involves limited transmembrane movement. Nature 436:852-856.

Chanda B, Blunck R, Faria LC, Schweizer FE, Mody I, Bezanilla F (2005b) A hybrid approach to measuring electrical activity in genetically specified neurons. Nat Neurosci 8:1619-1626.

Chen I, Howarth M, Lin W, Ting AY (2005) Site-specific labeling of cell surface proteins with biophysical probes using biotin ligase. Nat Methods 2:99-104

DiFranco M, Capote J, Quinonez M, Vergara JL (2007) Voltage-dependent dynamic FRET signals from the transverse tubules in mammalian skeletal muscle fibers. J Gen Physiol 130:581-600.

Domanov YA, Gorbenko GP (2002) Analysis of resonance energy transfer in model membranes: role of orientational effects. Biophys Chem 99:143-154.

Fernandez JM, Taylor RE, Bezanilla F (1983) Induced capacitance in the squid giant axon. Lipophilic ion displacement currents. J Gen Physiol 82:331-346.

Fisher JA, Barchi JR, Welle CG, Kim GH, Kosterin P, Obaid AL, Yodh AG, Contreras D, Salzberg BM (2008) Two-photon excitation of potentiometric probes enables optical recording of action potentials from mammalian nerve terminals in situ. J Neurophysiol 99:1545-1553.

Fung BK, Stryer L (1978) Surface density determination in membranes by fluorescence energy transfer. Biochemistry 17:5241-5248.

Gaboriaud R (1966) Sur le compartement des acides non chargés dans les milieux eau-méthanol. C R Acad Sci Ser C 263:991.

Gonzalez JE, Tsien RY (1995) Voltage sensing by fluorescence resonance energy transfer in single cells. Biophys J 69:1272-1280.

Gonzalez JE, Tsien RY (1997) Improved indicators of cell membrane potential that use fluorescence resonance energy transfer. Chem Biol 4:269-277.

Griffin BA, Adams SR, Tsien RY (1998) Specific covalent labeling of recombinant protein molecules inside live cells. Science 281:269-272.

Guerrero G, Siegel MS, Roska B, Loots E, Isacoff EY (2002) Tuning FlaSh: redesign of the dynamics, voltage range, and color of the genetically encoded optical sensor of membrane potential. Biophys J 83:3607-3618.

Harris KD (2005) Neural signatures of cell assembly organization. Nat Rev Neurosci 6:399-407.

Harris KD, Csicsvari J, Hirase H, Dragoi G, Buzsaki G (2003) Organization of cell assemblies in the hippocampus. Nature 424:552-556.

Harrison KH, Hof PR, Wang SS (2002) Scaling laws in the mammalian neocortex: does form provide clues to function? J Neurocytol 31:289-298. 
Iyer V, Hoogland TM, Saggau P (2006) Fast functional imaging of single neurons using random-access multiphoton (RAMP) microscopy. J Neurophysiol 95:535-545.

König P, Engel AK, Singer W (1996) Integrator or coincidence detector? The role of the cortical neuron revisited. Trends Neurosci 19:130-137.

Kralik JD, Dimitrov DF, Krupa DJ, Katz DB, Cohen D, Nicolelis MA (2001) Techniques for long-term multisite neuronal ensemble recordings in behaving animals. Methods 25:121-150.

Mainen ZF, Maletic-Savatic M, Shi SH, Hayashi Y, Malinow R, Svoboda K (1999) Two-photon imaging in living brain slices. Methods 18:231-239, 181.

Marks KM, Nolan GP (2006) Chemical labeling strategies for cell biology. Nat Methods 3:591-596.

Miesenböck G, Kevrekidis IG (2005) Optical imaging and control of genetically designated neurons in functioning circuits. Annu Rev Neurosci 28:533-563.

Miesenböck G, De Angelis DA, Rothman JE (1998) Visualizing secretion and synaptic transmission with $\mathrm{pH}$-sensitive green fluorescent proteins. Nature 394:192-195.

Murata Y, Iwasaki H, Sasaki M, Inaba K, Okamura Y (2005) Phosphoinositide phosphatase activity coupled to an intrinsic voltage sensor. Nature 435:1239-1243.

Nadeau H, McKinney S, Anderson DJ, Lester HA (2000) ROMK1 (Kir1.1) causes apoptosis and chronic silencing of hippocampal neurons. J Neurophysiol 84:1062-1075.

Ng M, Roorda RD, Lima SQ, Zemelman BV, Morcillo P, Miesenböck G (2002) Transmission of olfactory information between three populations of neurons in the antennal lobe of the fly. Neuron 36:463-474.

Nitabach MN, Blau J, Holmes TC (2002) Electrical silencing of Drosophila pacemaker neurons stops the free-running circadian clock. Cell 109:485-495.

Obaid AL, Koyano T, Lindstrom J, Sakai T, Salzberg BM (1999) Spatiotemporal patterns of activity in an intact mammalian network with single-cell resolution: optical studies of nicotinic activity in an enteric plexus. J Neurosci 19:3073-3093.

Ohki K, Chung S, Ch'ng YH, Kara P, Reid RC (2005) Functional imaging with cellular resolution reveals precise micro-architecture in visual cortex. Nature 433:597-603.

Ormö M, Cubitt AB, Kallio K, Gross LA, Tsien RY, Remington SJ (1996) Crystal structure of the Aequorea victoria green fluorescent protein. Science 273:1392-1395.

Oron D, Tal E, Silberberg Y (2005) Scanningless depth-resolved microscopy. Optics Express 13:1468-1476.

Perozo E, MacKinnon R, Bezanilla F, Stefani E (1993) Gating currents from a nonconducting mutant reveal open-closed conformations in Shaker K+ channels. Neuron 11:353-358.

Pu SC, Yang MJ, Hsu CC, Lai CW, Hsieh CC, Lin SH, Cheng YM, Chou PT (2006) The empirical correlation between size and two-photon absorption cross section of CdSe and CdTe quantum dots. Small 2:1308-1313.

Reddy GD, Saggau P (2005) Fast three-dimensional laser scanning scheme using acousto-optic deflectors. J Biomed Opt 10:064038.

Reiff DF, Ihring A, Guerrero G, Isacoff EY, Joesch M, Nakai J, Borst A (2005) In vivo performance of genetically encoded indicators of neural activity in flies. J Neurosci 25:4766-4778.

Rizzo MA, Springer GH, Granada B, Piston DW (2004) An improved cyan fluorescent protein variant useful for FRET. Nat Biotechnol 22:445-449.

Roorda RD, Hohl TM, Toledo-Crow R, Miesenböck G (2004) Video-rate nonlinear microscopy of neuronal membrane dynamics with genetically encoded probes. J Neurophysiol 92:609-621.

Sakai R, Repunte-Canonigo V, Raj CD, Knöpfel T (2001) Design and characterization of a DNA-encoded, voltage-sensitive fluorescent protein. Eur J Neurosci 13:2314-2318.

Salome R, Kremer Y, Dieudonne S, Leger JF, Krichevsky O, Wyart C, Chatenay D, Bourdieu L (2006) Ultrafast random-access scanning in two- photon microscopy using acousto-optic deflectors. J Neurosci Methods 154:161-174.

Salzberg BM, Davila HV, Cohen LB (1973) Optical recording of impulses in individual neurones of an invertebrate central nervous system. Nature 246:508-509.

Segall DE, Nelson PC, Phillips R (2006) Volume-exclusion effects in tethered-particle experiments: bead size matters. Phys Rev Lett 96:088306.

Shang Y, Claridge-Chang A, Sjulson L, Pypaert M, Miesenböck G (2007) Excitatory local circuits and their implications for olfactory processing in the fly antennal lobe. Cell 128:601-612.

Siegel MS, Isacoff EY (1997) A genetically encoded optical probe of membrane voltage. Neuron 19:735-741.

Sjulson L (2007) Two photon imaging of a genetically encodable voltage sensor, pp 31-33. New York: Cornell University.

Sjulson L, Miesenböck G (2007) Optical recording of action potentials and other discrete physiological events: a perspective from signal detection theory. Physiology (Bethesda) 22:47-55.

Smetters D, Majewska A, Yuste R (1999) Detecting action potentials in neuronal populations with calcium imaging. Methods 18:215-221.

Spruston N, Johnston D (1992) Perforated patch-clamp analysis of the passive membrane properties of three classes of hippocampal neurons. J Neurophysiol 67:508-529.

Swaminathan R, Hoang CP, Verkman AS (1997) Photobleaching recovery and anisotropy decay of green fluorescent protein GFP-S65T in solution and cells: cytoplasmic viscosity probed by green fluorescent protein translational and rotational diffusion. Biophys J 72:1900-1907.

Szabo G (1977) Electrical characteristics of ion transport in lipid bilayer membranes. Ann NY Acad Sci 303:266-280.

Thompson NL, Burghardt TP, Axelrod D (1981) Measuring surface dynamics of biomolecules by total internal reflection fluorescence with photobleaching recovery or correlation spectroscopy. Biophys J 33:435-454.

Thomson DJ (1982) Spectrum estimation and harmonic analysis. Proc IEEE 70:1055-1096.

Tsien RY, Gonzalez JE (2002) Detection of transmembrane potentials by optical methods. U.S. Patent 173:130.

Victor JD, Purpura KP (1996) Nature and precision of temporal coding in visual cortex: a metric-space analysis. J Neurophysiol 76:1310-1326.

Vucinic D, Bartol Jr TM, Sejnowski TJ (2006) Hybrid reflecting objectives for functional multiphoton microscopy in turbid media. Opt Lett 31:2447-2449.

Wang CC, Bruner LJ (1978) Lipid-dependent and phloretin-induced modifications of dipicrylamine adsorption by bilayer membranes. Nature 272:268-270.

Whalen AD (1971) Detection of signals in noise. New York: Academic.

Windisch B, Bray D, Duke T (2006) Balls and chains-a mesoscopic approach to tethered protein domains. Biophys J 91:2383-2392.

Wolber PK, Hudson BS (1979) An analytic solution to the Forster energy transfer problem in two dimensions. Biophys J 28:197-210.

Wong AM, Flores J, Wang JW (2005) Imaging olfactory activity in Drosophila CNS with a calcium-sensitive green fluorescent protein. In: Imaging in neuroscience and development: a laboratory manual (Yuste R, Konnerth A, eds). Cold Spring Harbor: Cold Spring Harbor Laboratory.

Yaksi E, Friedrich RW (2006) Reconstruction of firing rate changes across neuronal populations by temporally deconvolved $\mathrm{Ca} 2+$ imaging. Nat Methods 3:377-383.

Yang F, Moss LG, Phillips Jr GN (1996) The molecular structure of green fluorescent protein. Nat Biotechnol 14:1246-1251.

Zacharias DA, Violin JD, Newton AC, Tsien RY (2002) Partitioning of lipidmodified monomeric GFPs into membrane microdomains of live cells. Science 296:913-916.

Zipfel WR, Williams RM, Webb WW (2003) Nonlinear magic: multiphoton microscopy in the biosciences. Nat Biotechnol 21:1369-1377. 